\title{
Mixed finite element approximations of a singular elliptic problem based on some anisotropic and $h p$-adaptive curved quarter-point elements
}

\author{
Denise de Siqueira - denisesiqueira@utfpr.edu.br \\ Departamento de Matemática, UTFPR, Curitiba, PR, Brazil \\ Agnaldo M. Farias- agnaldo@gmail.com \\ Departamento de Matemática, IFNMG, Salinas, MG, Brazil \\ Phillipe R.B. Devloo - phil@fec.unicamp.br \\ FEC-Universidade Estadual de Campinas, Campinas-SP, Brazil \\ Sônia M. Gomes - soniag@ime.unicamp.br \\ IMECC-Universidade Estadual de Campinas, Campinas, SP, Brazil
}

\begin{abstract}
Mixed finite element methods are applied to a Poisson problem with singularity at a boundary point. The approximation spaces are based on quarter-point elements, the shape functions inheriting the singular behavior of their quadratic geometric maps. Two mesh scenarios are considered, by fixing some macro quarterpoint elements at the coarse level, and subdividing them by mapping uniformly refined square meshes on the master element by their corresponding geometric transforms. For eight-noded coarse quadrilateral quarter-point elements, placing two mid-side nodes near the singular vertex, radial singularity is exactly captured along element edges, and their refinements reveal shape regular curved meshes. For an improved version, using collapsed quadrilateral quarter-point elements obtained by reducing one of the quadrilateral element edges to the singular point, the radial singularity is captured inside the coarse macro elements as well. Their uniform refinement generates anisotropic meshes, grading towards the singular point. The assembly of the required $\mathbf{H}$ (div)-conforming approximation spaces based on these kind of meshes are described. Results for a typical test problem demonstrate a superior effectiveness of the proposed techniques for convergence acceleration, when confronted with usual affine finite elements, for $h, p$ and $h p$ adaptive refinements. Specially, collapsed quarter-point elements applied to the singular problem reveal accuracy rates equivalent to standard regular contexts, of smooth solutions discretized on uniform affine meshes.
\end{abstract}

Keywords: Mixed finite elements, $h p$ adaptivity, curved and anisotropic meshes, quarter-point elements, boundary point singularity. 


\section{Introduction}

A source of difficulties in the numerical simulation of elliptic problems in bounded domains is the occurrence of singularities in the solution. For instance, they can be caused by the presence of re-entrant boundary corners (or by abrupt change in boundary conditions), typical situations in fracture mechanics applications, where a radial square root singularity is representative for modeling the state of tension around a fracture in elasticity formulations.

Since the beginning of finite element history, poor convergence of standard $H^{1}$ conforming methods based on regular grids has been verified in the presence of this kind of low regularity behavior. One popular remedy for this drawback consists in adding special functions to the finite element approximation space, as early described in the papers [1]. This is also the principle guiding the extended finite element method $[2,3,4,5]$, using the notion of partition of unity [6]. Another different concept is the application of isoparametric mappings, with singular map for the geometry and for the construction of shape functions, as described in [7, 8]. In this context, the quarterpoint element is widely used in linear elastic fracture computations with $H^{1}$-conforming finite element methods. Adaptive numerical treatment of singular problems, incorporating local mesh refinement ( $h$-adaptivity) and/or different orders of approximating polynomials on separate elements in the same mesh ( $p$-adaptivity), has proved to be very effective $[9,10,11]$. Anisotropic mesh refinement has also been considered and analyzed in $[12,13,14,15]$, with improved results. There are also the so called Mapped Finite Element Methods (MFEM), initially proposed in [16], for better approximations of straight crack problems, and recently extended in [17] for more general crack configurations. Namely, the method can be interpreted as a reformulation of the problem statement in a reference parametric domain $\hat{\Omega}$, over which the singularity is eliminated, the corresponding parametric solution $\hat{u}$ being smoother than the original targeted one, and thus being able to be accurately solved using approximations based on standard uniform partitions of $\hat{\Omega}$. Similar ideas have been used for the construction of the Method of Auxiliary Mapping (MAM), summarized in [18], which has been applied for $p$ and $h p$-versions of various elliptic and elasticity problems.

The developed method incorporates all these aspects in some extent, but the adopted formulation is of mixed methods, where two variables are simulated simultaneously: the primal (potential) variable $u$, approximated by discontinuous functions in $L^{2}(\Omega)$, and the dual (flux) variable $\boldsymbol{\sigma}$, represented in $\mathbf{H}$ (div)-conforming spaces [19]. The interest is to evaluate the performance of this method for the resolution of a square-root radial point singularity by the incorporation of approximations based on quarter-point elements in the vicinity of the singularity point, the shape functions inheriting the singular behavior of the geometric map. As far as we understand, mixed formulations based in quarter-point elements have not been explored in the literature.

Two kinds of quarter-point elements shall be considered: the eight-noded quadrilateral element, placing two mid-side nodes near the singular vertex, and the collapsed quadrilateral quarter-point element, obtained by reducing one of the quadrilateral element edges to the singularity point, resulting a six node triangular element. The square-root radial singularity is exactly captured along element edges by the first type of quarter-point element, while in the latter case, it is captured inside the coarse macro 
elements as well. Starting from fixed macro quarter-point elements at the coarse level, they are refined by mapping uniformly refined square meshes on the master element by the corresponding geometric transforms. This uniform refinement reveals shape regular curved meshes inside coarse eight-noded quarter-point elements, and anisotropic meshes inside the coarse collapsed quadrilateral coarse elements, grading towards the singular point. In the later case, the meshes are formed by some triangles sharing the point singularity, and trapezoidal quadrilateral elements elsewhere.

As shall be illustrated by some verification tests, the proposed formulation based on quarter-point geometry share some underlying ideas of MFEM and MAM methods, in the sense that the method can be interpreted as local reformulation of the problem statement on the macro elements of the coarse mesh in the reference parametric domain $\hat{K}$, over which the singularity is eliminated, the corresponding parametric solution $\hat{u}$ being smoother than the original targeted one, and thus being able to be accurately solved using approximations based meshes uniformly refined. This effect has a great impact on simulations for the collapsed quadrilateral quarter-point scenario, but it has some limitations for eight-noded quadrilateral ones. For this later case, $h p$-adaptive meshes are employed for the quarter-point geometry in order to accelerate the convergence of the resulting approximate solutions.

Several methods have been developed for the construction of divergence conforming approximation spaces to be applied in flux approximations of the mixed element formulation. As described in [19, 20], they are usually formed by locally backtracking vector polynomial spaces restricted to the master element by the Piola transformation, which is defined in terms of the geometric map. The constructions of hierarchical high order spaces in $[21,22,23]$ are based on the properties of the De Rham complex. For the applications of the present paper, $\mathbf{H}(\mathrm{div})$-conforming finite element subspaces based on curved quadrilateral meshes, with $h p$-adaptation, are constructed. The vector shape functions form a hierarchy of bases, as proposed in [24] for triangular and quadrilateral affine elements, and extended to three-dimensional tetrahedral, hexahedral and prismatic affine meshes in [25]. Cases of $h p$-adaptive mixed formulations for other kinds of elements in two- and three-dimensional problems, including curved and surface elements, have been treated in [26, 27].

The paper is organized as follows. Some basic facts about the mixed element formulation for elliptic problems are described in Section 2. General aspects on the construction of $\mathbf{H}$ (div)-conforming approximation spaces for $h p$-adapted curved meshes, and the kinds of adopted approximation space configurations, are recalled in Section 3. Some properties of approximations based on quarter-point geometry are illustrated in Section 4. Applications of these space configurations for the mixed formulation of a model problem are discussed in Section 5 , for uniform and $h p$-adaptive frameworks. The results are confronted with the corresponding ones given by simulations based on affine square meshes, showing the dramatic error reduction when quarter-point elements are used. Section 6 gives the final conclusions.

The implementations are performed in the NeoPZ ${ }^{1}$ computational platform, which is an open-source object-oriented project providing a comprehensive set of high performance tools for finite element simulations, including the ones required for the present numerical study, namely $\mathbf{H}$ (div)-conforming approximation spaces, $h p$ adaptivity, curved

\footnotetext{
${ }^{1}$ http://github.com/labmec/neopz
} 
elements, and appropriate integration rules for functions showing point singularities.

\section{Mixed finite element formulation}

Consider a model Poisson problem:

$$
\begin{aligned}
\boldsymbol{\sigma} & =-\nabla u \text { in } \Omega, \\
\nabla \cdot \boldsymbol{\sigma} & =f \text { in } \Omega, \\
u & =u_{d} \text { on } \Gamma_{D}, \\
\nabla u \cdot \boldsymbol{\eta} & =g \text { on } \Gamma_{N},
\end{aligned}
$$

where $\partial \Omega=\Gamma_{D} \cup \Gamma_{N}, \Gamma_{D} \cup \Gamma_{N}=\emptyset, \Gamma_{D}$ and $\Gamma_{N}$ denoting the parts of $\partial \Omega$ for the enforcement of Dirichlet and Neumann boundary conditions, respectively, and $\boldsymbol{\eta}$ is the outward unit normal to $\partial \Omega$.

This problem can be expressed in the form: to find $u \in U=L^{2}(\Omega)$ and $\boldsymbol{\sigma} \in$ $\mathbf{V}=\left\{\mathbf{q} \in \mathbf{H}(\operatorname{div}, \Omega):\left.\mathbf{q} \cdot \boldsymbol{\eta}\right|_{\Gamma_{N}}=-g\right\}$, such that $\forall \varphi \in U$ and $\forall \mathbf{q} \in \mathbf{H}(\operatorname{div}, \Omega)$, with $\left.\mathbf{q} \cdot \boldsymbol{\eta}\right|_{\Gamma_{N}}=0$

$$
\begin{aligned}
a(\boldsymbol{\sigma}, \mathbf{q})+b(\mathbf{q}, u) & =c(\mathbf{q}), \\
b(\boldsymbol{\sigma}, \varphi) & =\ell(\varphi)
\end{aligned}
$$

where

$$
\begin{array}{r}
a(\boldsymbol{\sigma}, \mathbf{q})=\int_{\Omega} \boldsymbol{\sigma} \cdot \mathbf{q} d \Omega, \quad b(\mathbf{q}, u)=-\int_{\Omega} u \nabla \cdot \mathbf{q} d \Omega, \\
c(\mathbf{q})=-\int_{\Gamma_{d}} u_{d} \mathbf{q} \cdot \boldsymbol{\eta} d s, \quad \ell(\varphi)=-\int_{\Omega} f \varphi d \Omega .
\end{array}
$$

In typical $\mathbf{H}$ (div)-conforming discretized versions of this formulation, approximate solutions $\boldsymbol{\sigma}_{\mathcal{T}}$ and $u_{\mathcal{T}}$ are searched in finite dimensional spaces $\mathbf{V}_{\mathcal{T}} \subset \mathbf{V}$ and $U_{\mathcal{T}} \subset U$, and the weak formulation (1) is enforced with test functions in the corresponding spaces $\varphi \in U_{\mathcal{T}}$ and $\mathbf{q} \in \mathbf{V}_{\mathcal{T}}$, with vanishing normal components over $\partial \Omega$.

The natural functional context for the analysis of the mixed formulation (1), and of its discretized versions, is of saddle-point problems, for which the celebrated inf-sup condition (also known as LBB condition, for Ladyženskaja-Babuška-Brezzi) is necessary and sufficient for well-posedness determination. The verification of the inf-sup condition requires a careful choice of the approximation spaces $\mathbf{V}_{\mathcal{T}}$ and $U_{\mathcal{T}}$, by enforcing the property $\nabla \cdot \hat{\mathbf{V}}=\hat{U}$ of the associated spaces on the master element. This fact allows the definition of a projection $\hat{\boldsymbol{\pi}}$ over $\hat{\mathbf{V}}$ commuting the de Rham diagram

$$
\begin{array}{ccc}
\mathbf{H}^{\alpha}(\Omega) & \stackrel{\nabla \cdot}{\longrightarrow} & L^{2}(\Omega) \\
\downarrow \hat{\boldsymbol{\pi}} & & \downarrow \hat{\lambda} \\
\hat{\mathbf{V}} & \stackrel{\nabla \cdot}{\longrightarrow} & \hat{U}
\end{array}
$$

where $\hat{\lambda}$ is the usual $L^{2}$-projection over $\hat{U}$. These projections are globally extended to $\Pi_{\mathcal{T}}$ over $\mathbf{V}_{\mathcal{T}}$, and $\Lambda_{\mathcal{T}}$ over $U_{\mathcal{T}}$.

For such stable finite element approximation spaces, the following estimates hold for the approximate solutions $\boldsymbol{\sigma}_{\mathcal{T}} \in \mathbf{V}_{\mathcal{T}}$ and $u_{\mathcal{T}} \in U_{\mathcal{T}}[19,28]$ :

$$
\left\|\boldsymbol{\sigma}-\boldsymbol{\sigma}_{\mathcal{T}}\right\|_{L^{2}} \lesssim\left\|\boldsymbol{\sigma}-\boldsymbol{\Pi}_{\mathcal{T}} \boldsymbol{\sigma}\right\|_{L^{2}}
$$




$$
\begin{aligned}
\left\|\nabla \cdot\left(\boldsymbol{\sigma}-\boldsymbol{\sigma}_{\mathcal{T}}\right)\right\|_{L^{2}} & \lesssim\left\|\nabla \cdot\left(\boldsymbol{\sigma}-\boldsymbol{\Pi}_{\mathcal{T}} \boldsymbol{\sigma}\right)\right\|_{L^{2}} \\
\left\|u-u_{\mathcal{T}}\right\|_{L^{2}} & \lesssim\left\{\left\|\boldsymbol{\sigma}-\boldsymbol{\Pi}_{\mathcal{T}} \boldsymbol{\sigma}\right\|_{L^{2}}+\left\|u-\Lambda_{\mathcal{T}} u\right\|_{L^{2}}\right\} .
\end{aligned}
$$

We also remark that, assuming elliptic-regularity (verified by convex polygons or smooth regions $\Omega$ ), and using duality arguments, enhanced order of accuracy can be obtained for $u_{\mathcal{T}}$ when enriched space configurations are used, as in the case of $R T_{k}^{+}$ spaces described next.

Projection convergence rates in $L^{2}$-norms for these finite element approximations are usually determined by the largest total degree of polynomials included in the local spaces $\mathbf{V}(K)=\mathbb{F}_{K}^{d i v} \hat{\mathbf{V}}$ and $\hat{U}(K)=\mathbb{F}_{K}(\hat{U})$. For instance, assuming shape regular meshes $\mathcal{T}_{h}$, with maximum element diameters $h$, and uniform polynomial distribution all over the elements, if $k$ and $t$ are the largest natural numbers such that $\left[\mathbb{P}_{k}\right]^{2} \subset \mathbf{V}(K)$, and $\mathbb{P}_{t} \subset U(K)$, then for $\boldsymbol{\sigma} \in \mathbf{H}^{q}(\Omega)$ and $u \in H^{s}(\Omega)$, with integer indices $0<q \leq k+1$, and $0<s \leq t+1$, the next estimations hold for approximations based on affine and for bilinearly mapped elements [19, 20]

$$
\begin{aligned}
\left\|\boldsymbol{\sigma}-\boldsymbol{\Pi}_{h} \boldsymbol{\sigma}\right\|_{L^{2}(\Omega)} & \lesssim h^{q+1}|\boldsymbol{\sigma}|_{\mathbf{H}^{k+1}(\Omega)}, \\
\left\|u-\Lambda_{h}\right\|_{L^{2}} & \lesssim h^{t+1}|u|_{H^{s+1}(\Omega)},
\end{aligned}
$$

the constants arising in the error estimates being depend on the ratio between the outer and inner diameter of the elements.

In [29], Corollary 5.3, quasi-interpolation operators have been constructed for which similar estimations hold for the cases of fractional Sobolev indices $q$ and $s$, for affine meshes. Consequently, high order approximation spaces based on affine meshes have limited usefulness for problems with corner sigularities, with slow convergence determined by the low regularity of the solution $u \in H^{s}(\Omega), 0<s<1$. For such problems, methods using anisotropic mesh grading (with aspect ratio of elements growing to infinity in a refined region close to the singularity) are capable of achieving better accuracy. But for such frameworks, the above mentioned error analysis does not hold. The works in $[13,14]$ are dedicated to the study of interpolation properties of RaviartThomas elements for some specific anisotropic affine meshes, which are meant for the approximation of this kind of singular solutions. In [13], error estimates for the mixed formulation for approximations based on anisotropic meshes are obtained, showing first order improvement.

For the current applications using quarter-point elements, the quadratic geometric mappings are not affine nor bilinear. However, the mapped approximation spaces inherit the singular behavior of the geometric map, which is in accordance with the targeted problem solution. As shall be verified, due to the adopted particular refinement procedure, by a global mapping of uniform partitions on the master element, the resulting meshes based on eight-noded quadrilateral quarter-point elements are shape regular, and graded anisotropic meshes occur for collapsed quadrilateral quarter point elements. 


\section{Space configurations based on $h, p$ and $h p$-adaptive curved meshes}

Let $\Omega \subset \mathbb{R}^{2}$ be a Lipschitz computational domain, with boundary $\partial \Omega$, and denote by $\boldsymbol{\eta}$ its outward unit normal. Let $\mathcal{T}$ be a partition of $\Omega$ formed by elements $K$. We shall be concerned with elements mapped from rectangular master element by quarter-point quadratic geometric transforms, and $\mathcal{T}$ does not have, in principle, any limitation on hanging sides and approximation order distribution $\mathbf{k}=\left(k_{K}\right)$.

Approximation vector spaces

$$
\mathbf{V}_{\mathcal{T}}^{\mathbf{k}} \subset \mathbf{H}(\operatorname{div}, \Omega)=\left\{\mathbf{q} \in L^{2}(\Omega)^{2} ; \nabla \cdot \mathbf{q} \in L^{2}(\Omega)\right\},
$$

which are defined piecewise over the elements of $\mathcal{T}$, require that the local pieces $\mathbf{q}_{K}=\left.\mathbf{q}\right|_{K}$ should be assembled by keeping continuous normal components across common element edges. For potential approximations, scalar spaces $U_{\mathcal{T}}^{\mathbf{k}} \subset L^{2}(\Omega)$ are also piecewise defined over the elements of $\mathcal{T}$, but without any continuity constraint across element interfaces.

\subsection{Construction of hp-adaptative spaces}

The adoted methodology for the assembly of $h p$-adaptive $\mathbf{H}$ (div)-conforming spaces based on curved meshes has been proposed in [27] for three dimensional problems. It follows a sequence of steps.

1. To each element $K$, there is a bijective geometric mapping $F_{K}: \hat{K} \rightarrow K$, associating each point $(\xi, \eta) \in \hat{K}$ of the rectangular master element $\hat{K}$ to a point $(x, y)=F_{K}(\xi, \eta) \in K$. Usually, it is assumed that $F_{K}$ is differentiable, and that the determinant of its Jacobian matrix does not vanish. An isomorphism $\mathbb{F}_{K}$ : $\hat{\varphi} \rightarrow \varphi$, mapping scalar functions of $H^{1}(\hat{K})$ to scalar functios of $H^{1}(K)$, is induced by the geometric mapping. It also induces a contravariant Piola transformation $\mathbb{F}_{K}^{d i v}: \hat{\mathbf{q}} \rightarrow \mathbf{q}$, an isomorphism mapping vector fields $\hat{\mathbf{q}} \in \mathbf{H}(\operatorname{div}, \hat{K})$ to vector fields $\mathbf{q} \in \mathbf{H}(\operatorname{div}, K)$. It is defined by the formula

$$
\mathbf{q}=\mathbb{F}_{K}^{d i v} \hat{\mathbf{q}}=\mathbb{F}_{K}\left[\frac{1}{\mathbf{J}_{K}} D F_{K} \hat{\mathbf{q}}\right]
$$

where $D F_{K}$ is the Jacobian of the geometric mapping $F_{K}$, and $J_{K}=\operatorname{det} D F_{K}$. There is also an isomorphism $\mathbb{G}: \hat{\mu} \rightarrow \mu$ mapping functions $\hat{\mu} \in H^{1 / 2}(\partial \hat{K})$ to functions $\mu \in H^{1 / 2}(\partial K)$ such that $\mu=\mathbb{F}_{K}\left[\frac{1}{J_{\mathbf{n}}} \hat{\mu}\right], \mathbf{J}_{\mathbf{n}}=J_{K}{ }^{t} D F_{K}^{-1} \hat{\mathbf{n}} \mid,{ }^{t} D F_{K}^{-1}$ being the transpose of the inverse of $D F_{K}$. Throughout this paper, $\mathbf{n}$ denotes the outward unit normal to the faces of an element $K \in \mathcal{T}$.

2. Vector polynomial spaces $\hat{\mathbf{V}}_{k_{K}}$ are defined in $\hat{K}$. For the cases to be considered, the parameter $k_{K}$ refers to the maximum degree of the normal components $\hat{\mathbf{q}}$. $\left.\hat{n}\right|_{\partial \hat{K}}$, and to the full polynomial space $\left[\mathbb{Q}_{k(K), k(K)}\right]^{2}$ contained in $\hat{\mathbf{V}}_{k_{K}}$.

3. A family of hierarchical bases $\mathbf{B}_{k_{K}}^{\hat{K}}=\{\hat{\boldsymbol{\Phi}}\}$ for $\hat{\mathbf{V}}_{k_{K}}$ is given, as proposed in [24]. The principle is to choose appropriate constant vector fields $\hat{\mathbf{v}}$, based on the geometry of the master element, which are multiplied by an available hierarchic 
set of $H^{1}$ scalar basic functions $\hat{\varphi}$ in order to get $\hat{\boldsymbol{\Phi}}=\hat{\varphi} \hat{\mathbf{v}}$. There are shape functions of interior type, with vanishing normal components over all element faces. Otherwise, the shape functions are classified of edge type. The normal component of an edge function coincides on the corresponding edge with the associated scalar shape function, and vanishes over the other edges.

4. A hierarchic family of vector bases $\mathbf{B}_{k_{K}}^{K}=\{\boldsymbol{\Phi}\}$ is defined over $K$ by the Piola transformation $\mathbf{\Phi}=\mathbb{F}_{K}^{d i v}(\hat{\mathbf{\Phi}})$. They inherit the classification of their counterparts on the master element: there are shape functions of interior type, with vanishing normal components over all element edges. Otherwise, $\boldsymbol{\Phi}$ is classified as of edge type, its normal component on the associated edge coinciding with the multiplication of the scalar shape function $\varphi=\mathbb{F}_{K} \hat{\varphi}$ by the normal component of the Piola mapped vector used in its definition, and vanishing over the other edges.

5. Approximation spaces $\mathbf{V}_{\mathcal{T}}^{\mathbf{k}} \subset \mathbf{H}(\operatorname{div}, \Omega)$ are formed by functions $\mathbf{q} \in\left[L^{2}(\Omega)\right]^{2}$, which are defined piecewise over the elements of $\mathcal{T}$ by local functions $\mathbf{q}_{K}=\left.\mathbf{q}\right|_{K} \in$ span $\mathbf{B}_{k_{K}}^{K} \subset \mathbf{H}(\operatorname{div}, K)$. They can be assembled to get continuous normal components on the elements interfaces. This property is obtained as a consequence of the particular properties verified by the proposed vector shape functions and of the continuity of the scalar shape functions used in their construction.

\subsection{Basic facts about the Piola transformation}

As already mentioned, the geometric mapping $F_{K}$ induces the contravariant Piola transformation $\mathbb{F}_{K}^{d i v}: \hat{\mathbf{q}} \rightarrow \mathbf{q}$, mapping vector-valued functions $\hat{\mathbf{q}} \in \mathbf{H}(\operatorname{div}, \hat{K})$ to mapping vector-valued functions $\boldsymbol{\Phi} \in \mathbf{H}(\operatorname{div}, K)$. Furthermore, if $\varphi=\mathbb{F}_{K} \hat{\varphi} \in H^{1}(K)$, the following identities are valid

$$
\begin{aligned}
\int_{K} \mathbf{q} \cdot \nabla \varphi d x d y & =\int_{\hat{K}} \hat{\mathbf{q}} \cdot \hat{\nabla} \hat{\varphi} d \xi d \eta \\
\int_{K} \varphi \nabla \cdot \mathbf{q} d x d y & =\int_{\hat{K}} \hat{\varphi} \hat{\nabla} \cdot \hat{\mathbf{q}} d \xi d \eta \\
\int_{\partial K} \mathbf{q} \cdot \mathbf{n} \varphi d s & =\int_{\partial \hat{K}} \hat{\mathbf{q}} \cdot \hat{\mathbf{n}} \hat{\varphi} d \hat{s}
\end{aligned}
$$

Property (7) says that the divergence of a transformed vector-valued function is given by the the expression

$$
\nabla \cdot \mathbf{q}=\mathbb{F}_{K}\left[\frac{1}{\mathbf{J}_{K}} \hat{\nabla} \cdot \hat{\mathbf{q}}\right]
$$

and property (8) means that the normal trace $\boldsymbol{\Phi} \cdot \mathbf{n}$ over $\partial K$ is the image by the mapping $\mathbb{G}$ of the normal trace $\hat{\mathbf{q}} \cdot \hat{\mathbf{n}}$ over $\partial \hat{K}$ :

$$
\left.\mathbf{q} \cdot \mathbf{n}\right|_{\partial K}=\left.\frac{1}{\mathbf{J}_{\mathbf{n}}} \hat{\mathbf{q}} \cdot \hat{\mathbf{n}}\right|_{\partial \hat{K}}
$$

Specific details for quarter-point maps shall be given in Section 4 . 


\subsection{Approximation space configurations}

Following the developments in $[25,30]$, we shall consider two stable configurations for approximation spaces to be used for primal $u$ and dual $\boldsymbol{\sigma}$ variables in discretized versions of the mixed formulation.

- The first case corresponds to the celebrated Raviart-Thomas configuration $R T_{\mathbf{k}}$, where for a given $k=k_{K}$, the polynomial space on the master element is $\hat{\mathbf{V}}_{R T_{k}}=\mathbb{Q}_{k+1, k} \times \mathbb{Q}_{k, k+1}$. The scalar space $\hat{U}_{R T_{k}}=\mathbb{Q}_{k, k}$ is chosen such that the compatibility property $\nabla \cdot \hat{\mathbf{V}}_{R T_{k}}=\hat{U}_{R T_{k}}$ is verified.

- Another type of space configuration, denoted by $R T_{\mathbf{k}}^{+}$, corresponds to an enriched version of $R T_{\mathbf{k}}$. The flux space $\hat{\mathbf{V}}_{R T_{k}^{+}}$is formed by including in $\hat{\mathbf{V}}_{R T_{k}}$ the internal shape functions of $\hat{\mathbf{V}}_{R T_{k+1}}$, while keeping the edge functions with normal components of degree $k$. The scalar primal approximations are also enriched to $\hat{U}_{R T_{k}^{+}}=\mathbb{Q}_{k+1, k+1}$, such that the compatibility property $\nabla \cdot \hat{\mathbf{V}}_{R T_{k}^{+}}=\hat{U}_{R T_{k}^{+}}$is kept.

For sufficiently smooth solutions, uniform polynomial degree distribution $\mathbf{k}(k)=k$, and shape regular rectangular meshes, it is well know that convergence rates for the mixed finite element approximations based on the $R T_{k}$ space configuration is of order $k+1$ for the flux variable, measured by the $\mathbf{H}(\mathrm{div})$-norm, and for $L^{2}$-errors of the potential variable. As shown in [30], for general bilinearly mapped quadrilateral elements, and for enriched $R T_{k}^{+}$space configurations, the flux error in $\mathbf{H}($ div)-norm is also of order $k+1$, and the potential accuracy enhances to order $k+2$ when the $\Omega$ is convex.

The computational effort in mixed formulations can be reduced by the application of static condensation, the condensed system being solved only for flux edge terms and a constant value for $u$ in each element, all internal fluxes and potential degrees of freedom, excepting a constant value in each element, are condensed. Thus, the dimension of the static condensed matrix coincides for the approximations spaces of types $R T_{\mathrm{k}}$ and $R T_{\mathrm{k}}^{+}$.

\section{Quarter-point geometry}

Following [7], let $\hat{K}=[-1,1] \times[-1,1]$ be the master element, and consider the nodes $\left(\xi_{i}, \eta_{i}\right) \in \hat{K}$ defined in the next Table 1 .

\begin{tabular}{|c|c|c|c|c|c|c|c|c|}
\hline$i$ & 1 & 2 & 3 & 4 & 5 & 6 & 7 & 8 \\
\hline coordinates & \multicolumn{5}{|c|}{ Vertices } & \multicolumn{4}{|c|}{ Mid-side nodes } \\
\hline \hline$\xi_{i}$ & -1 & 1 & 1 & -1 & 0 & 1 & 0 & -1 \\
\hline$\eta_{i}$ & -1 & -1 & 1 & 1 & -1 & 0 & 1 & 0 \\
\hline
\end{tabular}

Table 1: Vertices and mid-point nodes of the master element $\hat{K}$.

Let $N_{i}(\xi, \eta)$ be the quadratic Lagrange shape functions

$$
\begin{aligned}
N_{i}(\xi, \eta)= & {\left[\left(1+\xi \xi_{i}\right)\left(1+\eta \eta_{i}\right)-\left(1-\xi^{2}\right)\left(1+\eta \eta_{i}\right)-\left(1-\eta^{2}\right)\left(1+\xi \xi_{i}\right)\right] \frac{\xi_{i}^{2} \eta_{i}^{2}}{4} } \\
& +\left(1-\xi^{2}\right)\left(1+\eta \eta_{i}\right)\left(1-\xi_{i}^{2}\right) \frac{\eta_{i}^{2}}{2}+\left(1-\eta^{2}\right)\left(1+\xi \xi_{i}\right)\left(1-\eta_{i}^{2}\right) \frac{\xi_{i}^{2}}{2}
\end{aligned}
$$


at the nodes $\left(\xi_{i}, \eta_{i}\right)$. Precisely, for the vertices $\left(\xi_{i}, \eta_{i}\right)$, where $\xi_{i}^{2}=\eta_{i}^{2}=1$,

$$
N_{i}(\xi, \eta)= \begin{cases}\frac{1}{4}(1-\xi)(1-\eta)[-1-\xi-\eta], & \text { at node } 1, \\ \frac{1}{4}(1+\xi)(1-\eta)[-1+\xi-\eta], & \text { at node } 2, \\ \frac{1}{4}(1+\xi)(1+\eta)[-1+\xi+\eta], & \text { at node } 3, \\ \frac{1}{4}(1-\xi)(1+\eta)[-1-\xi+\eta], & \text { at node } 4\end{cases}
$$

For the nodes 5 and 7 , with $\xi_{i}^{2}=0$ and $\eta_{i}^{2}=1$,

$$
N_{i}(\xi, \eta)= \begin{cases}\frac{1}{2}\left(1-\xi^{2}\right)(1-\eta), & \text { at node } 5, \\ \frac{1}{2}\left(1-\xi^{2}\right)(1+\eta), & \text { at node } 7 .\end{cases}
$$

For the nodes 6 and 8 , with $\xi_{i}^{2}=1$ and $\eta_{i}^{2}=0$,

$$
N_{i}(\xi, \eta)= \begin{cases}\frac{1}{2}\left(1-\eta^{2}\right)(1+\xi), & \text { at node } 6 \\ \frac{1}{2}\left(1-\eta^{2}\right)(1-\xi), & \text { at node } 8\end{cases}
$$

Given a quadrilateral element $K$, with vertices $\left(x_{1}, y_{1}\right),\left(x_{2}, y_{2}\right),\left(x_{3}, y_{3}\right)$, and $\left(x_{4}, y_{4}\right)$, consider mid-side nodes $\left(x_{5}, y_{5}\right),\left(x_{6}, y_{6}\right),\left(x_{7}, y_{7}\right)$, and $\left(x_{8}, y_{8}\right)$ placed on the edges $\left(x_{1}, y_{1}\right)-\left(x_{2}, y_{2}\right),\left(x_{2}, y_{2}\right)-\left(x_{3}, y_{3}\right),\left(x_{3}, y_{3}\right)-\left(x_{4}, y_{4}\right)$, and $\left(x_{4}, y_{4}\right)-\left(x_{1}, y_{1}\right)$, respectively. Define $F_{K}: \hat{K} \rightarrow K$ by the formulae

$$
x(\xi, \eta)=\sum_{i=1}^{8} N_{i}(\xi, \eta) x_{i}, \quad y(\xi, \eta)=\sum_{i=1}^{8} N_{i}(\xi, \eta) y_{i}
$$

such that $\left(x_{i}, y_{i}\right)=F_{K}\left(\xi_{i}, \eta_{i}\right)$. According to [7], the name quarter-point element comes when two mid-point nodes, namely, $\left(x_{5}, y_{5}\right)$ and $\left(x_{8}, y_{8}\right)$, of the sides sharing a vertex, $\left(x_{1}, y_{1}\right)$ for this case, are moved towards it, at one fourth of their associated side length, while the other two mid-side nodes $\left(\left(x_{6}, y_{6}\right)\right.$ and $\left.\left(x_{7}, y_{7}\right)\right)$ are the middle points of the corresponding sides.

\subsection{Uniform subdivision of a quarter-point element}

Given a quarter-point element $K$ mapped by the quadratic transformation $F_{K}$, its refinement into four sub-elements $K_{j}$ results after the following steps:

1. Let $S_{j}$ be the four squares obtained by a uniform subdivision of $\hat{K}$, and consider affine transformations $L_{j}: \hat{K} \rightarrow \hat{S}_{j}$

2. The sub-elements in $K$ correspond to $K_{j}=F_{K} \hat{S}_{j}$.

3. The quadratic mappings $F_{K_{j}}: \hat{K} \rightarrow K_{j}$ associated to $K_{j}$ are defined by the composition $F_{K_{j}}=F_{K} \circ L_{j}$.

Therefore, the vertices of the resulting 4 curved sub-elements in $K$ are determined by the four vertices of $K$, namely $\left(x_{1}, y_{1}\right),\left(x_{2}, y_{2}\right),\left(x_{3}, y_{3}\right)$, and $\left(x_{4}, y_{4}\right)$, the four side nodes $\left(x_{5}, y_{5}\right),\left(x_{6}, y_{6}\right),\left(x_{7}, y_{7}\right)$, and $\left(x_{8}, y_{8}\right)$, and the central point $\left(x_{9}, y_{9}\right)=F_{K}(0,0)$. Note that

$$
N_{i}(0,0)=-\frac{\xi_{i}^{2} \eta_{i}^{2}}{4}+\left(1-\xi_{i}^{2}\right) \frac{\eta_{i}^{2}}{2}+\left(1-\eta_{i}^{2}\right) \frac{\xi_{i}^{2}}{2}
$$


giving $N_{i}(0,0)=-0.25$ for the vertices $i=1,2,3$ and 4 , and $N_{i}(0,0)=0.5$, for the mid-side nodes $i=5,6,7$ and 8 . Consequently

$$
\begin{aligned}
& x_{9}=-0.25 \sum_{i=1}^{4} x_{i}+0.5 \sum_{i=5}^{8} x_{i}, \\
& y_{9}=-0.25 \sum_{i=1}^{4} y_{i}+0.5 \sum_{i=5}^{8} y_{i} .
\end{aligned}
$$

\subsection{Example of quarter-point element with 8 nodes}

Suppose that $K=[0,0.5] \times[0,0.5]$, with vertices and mid-side nodes given in Table 2 , and illustrated in Figure 1.

\begin{tabular}{|c|c|c|c|c|c|c|c|c|}
\hline$i$ & 1 & 2 & 3 & 4 & 5 & 6 & 7 & 8 \\
\hline coordinates & \multicolumn{5}{|c|}{ Vertices } & \multicolumn{5}{c|}{ Mid-side nodes } \\
\hline \hline$x_{i}$ & 0 & 0.5 & 0.5 & 0 & 0.125 & 0.5 & 0.25 & 0 \\
\hline$y_{i}$ & 0 & 0 & 0.5 & 0.5 & 0 & 0.25 & 0.5 & 0.125 \\
\hline
\end{tabular}

Table 2: Example: vertices and mid-point nodes of a eight-noded quadrilateral quarterpoint element $K$.
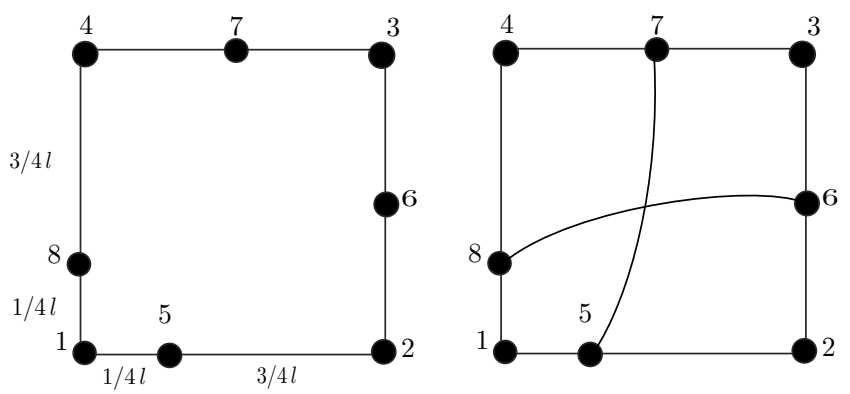

Figure 1: Example of quarter-poin-element $K$ : mid-side node movement towards the vertex $\left(x_{1}, y_{1}\right)$ to one quarter of the correspondind side length $l=0.5$ (left side), and the result of one subdivision of $K$ (rigth side).

For this element, the quarter-point map becomes

$$
x=\frac{1}{16}\left(3+4 \xi+\eta+\xi^{2}-\xi^{2} \eta\right), \quad y=\frac{1}{16}\left(3+\xi+4 \eta+\eta^{2}-\xi \eta^{2}\right) .
$$

Observe that, along the line $\eta=-1$, the result of the the quarter-point map gives $x=\frac{1}{8}(1+\xi)^{2}$, and $y=0$. That is, over the side $y=0$, the square root singular function $\sqrt{8 x}=1+\xi$ is reproduced in the master element side $\eta=-1$ by a first order polynomial. Take the more general square-root function $w(r, \theta)=\sqrt{r}=\left(x^{2}+y^{2}\right)^{\frac{1}{4}}$ restricted to $K$, as plotted in Figure 2 (left side). Its representation $\hat{w}(\xi, \eta)$ back in the master element $\hat{K}$, i.e., $\hat{w}(\xi, \eta)=w\left(F_{K}(\xi, \eta)\right)$, has a Taylor expansion around $(-1,-1)$ is given by

$$
\begin{aligned}
\hat{w}(\xi, \eta) & =\frac{\eta+1}{2 \sqrt{2}}+\left(\frac{1}{4 \sqrt{2}}-\frac{\eta+1}{8 \sqrt{2}}\right)(\xi+1)+\frac{1}{16 \sqrt{2}} \frac{(\xi+1)^{2}}{(\eta+1)} \\
& +O(\eta+1)^{3}+\mathcal{O}(\xi+1)^{3}+\mathcal{O}\left((\eta+1)^{3}(\xi+1)\right)+\mathcal{O}\left((\eta+1)^{3}(\xi+1)^{2}\right) .
\end{aligned}
$$


Its plot is shown in Figure 2 (right side), which looks much smoother than $w$, illustrating the capability of quarter-point map $F_{K}$ to well represent square-root type of singularities.
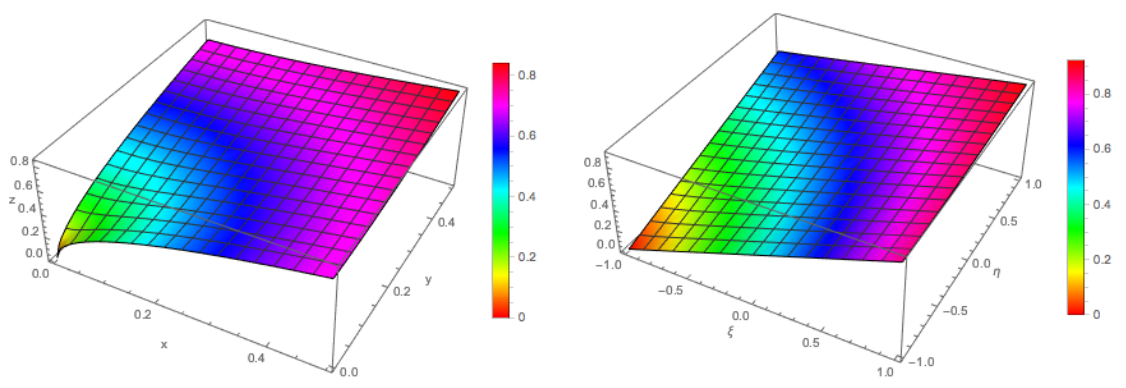

Figure 2: Example: $w(r, \theta)=\sqrt{r}$, restricted to $K$ (left side), and its parametric representation $\hat{w}(\xi, \eta)$ in the master element $(\xi, \eta) \in \hat{K}$.

\section{Piola transformation}

The Jacobian matrix of $F_{K}$ for this particular quarter-point element is

$$
D F_{K}=\left[\begin{array}{cc}
\frac{1}{4}+\frac{1}{8} \xi(1-\eta) & \frac{1}{16}+\frac{1}{16} \eta^{2} \\
\frac{1}{16}\left(1-\eta^{2}\right) & \frac{1}{4}+\frac{1}{8} \eta(1-\xi)
\end{array}\right] .
$$

Note that, over the side $y=0, \frac{\partial x}{\partial \xi}=\frac{1}{4}(1+\xi)=\frac{1}{4} \sqrt{8 x}$, making the Jacobian singular at the vertex $\left(x_{1}, y_{1}\right)=(0,0)$.

By the Piola transformation $\mathbf{q}=\mathbb{F}_{K}^{d i v} \hat{\mathbf{q}}$, the parametrized version $\hat{\mathbf{q}}$ associated to a vector function $\mathbf{q}$ has the formula

$$
\hat{\mathbf{q}}(\xi, \eta)=J_{K}(\hat{\mathbf{x}}) D F_{K}(\xi, \eta)^{-1} \mathbf{q}=\left[\begin{array}{cc}
\frac{\partial y}{\partial \eta} & -\frac{\partial x}{\partial \eta} \\
-\frac{\partial y}{\partial \xi} & \frac{\partial x}{\partial \xi}
\end{array}\right] \mathbf{q} .
$$

Some examples of parametrized vector functions are shown in Table 3 for this particular eight-noded quadrilateral quarter point element $K$. Observe that, for $\mathbf{q}=[1,0]^{t}$ and $\mathbf{q}=[0,1]^{t}, \hat{\mathbf{q}} \in \hat{\mathbf{V}}_{R T_{1}}=\mathbb{Q}(2,1) \times \mathbb{Q}(1,2)$, implying that $\mathbf{V}_{R T_{1}}(K)$ contains the constant vectors.

\begin{tabular}{|c|c|c|}
\hline $\mathbf{q}$ & \multicolumn{2}{|c|}{$\hat{\mathbf{q}}$} \\
\cline { 2 - 3 } & Quadrilateral Q-P & Collapsed quadrilateral Q-P \\
\hline$\left[\begin{array}{l}1 \\
0\end{array}\right]$ & {$\left[\begin{array}{c}\frac{1}{4}+\frac{1}{8} \eta(1-\xi) \\
-\frac{1}{16}\left(1-\eta^{2}\right)\end{array}\right]$} & {$\left[\begin{array}{c}\frac{1}{8}(1+\xi)^{2} \\
-\frac{1}{4} \eta(1+\xi)\end{array}\right]$} \\
\hline$\left[\begin{array}{l}0 \\
1\end{array}\right]$ & {$\left[\begin{array}{c}-\frac{1}{16}+\frac{1}{16} \xi^{2} \\
\frac{1}{4}+\frac{1}{8} \xi(1-\eta)\end{array}\right]$} & {$\left[\begin{array}{c}0 \\
\frac{1}{4}(\xi+1)\end{array}\right]$} \\
\hline
\end{tabular}

Table 3: Parametrized vector functions $\hat{\mathbf{q}}$ associated to constant vector functions $\mathbf{q}$ defined in quadrilateral eight-noded and collapsed quadrilateral six-noded quarter-point elements $K$. 


\section{Uniform refinement of $K$}

Setting $\mathcal{T}_{0}^{K}=K$, a recursive application of the refinement procedure generates curved refined meshes $\mathcal{T}_{j}^{K}$ of the $K$ obtained as the image of the uniformly refined mesh $\mathcal{T}_{j}^{\hat{K}}$, with spacing $2^{-j}$, by the global quarter-point mapping $F_{K}$ of the macro-element $K$. The partition $\mathcal{T}_{1}^{K}$ of the first subdivision step for this particular example of quarterpoint element $K$ is illustrated on the right side of Figure 1, whose middle point is $\left(x_{9}, y_{9}\right)=F_{K}(0,0)=(0.1875,0.1875)$. Illustrations of $\mathcal{T}_{j}^{K}$, for $j=2,3$, are shown in Figure 3 (left side). It can be observed that the uniform refinement of the quarter-point element $K$ produces a directional refinement pattern pointing towards the singularity point $\left(x_{1}, x_{1}\right)$. Despite of their stretched aspect, the meshes $\mathcal{T}_{j}^{K}$ are shape-regular, as illustrated in Figure 6 (left side).
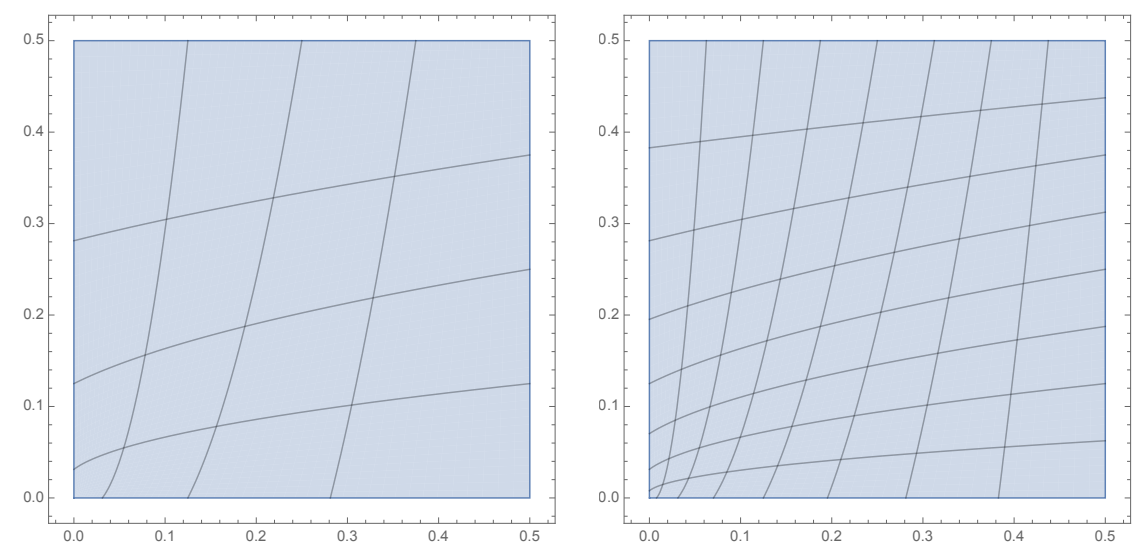

Figure 3: Meshes $\mathcal{T}_{j}^{K}, j=2,3$, obtained by uniform subdivisions of eight-noded quarter point element $K$.

\subsection{Collapsed quadrilateral quarter-point element}

Consider the node numbering on the master element in Table 4, as in [31].

\begin{tabular}{|c|c|c|c|c|c|c|c|c|}
\hline$i$ & 1 & 3 & 5 & 7 & 2 & 4 & 6 & 8 \\
\hline coordinates & \multicolumn{5}{|c|}{ Vertices } & \multicolumn{5}{|c|}{ Mid-points } \\
\hline \hline$\xi_{i}$ & -1 & 1 & 1 & -1 & 0 & 1 & 0 & -1 \\
\hline$\eta_{i}$ & -1 & -1 & 1 & 1 & -1 & 0 & 1 & 0 \\
\hline
\end{tabular}

Table 4: Numbering of vertices and mid-point nodes of the master element $\hat{K}$ [31].

Suppose that $K$ is the triangular element with six nodes shown in Table 5 , which can be interpreted by the collapse of the nodes 1 and 7 in the master element on the singular node 8 . 


\begin{tabular}{|c|c|c|c|c|c|c|c|c|}
\hline$i$ & 1 & 3 & 5 & 7 & 2 & 4 & 6 & 8 \\
\hline coordinates & \multicolumn{5}{|c|}{ Vertices } & \multicolumn{5}{|c|}{ Mid-points } \\
\hline \hline$x_{i}$ & 0 & 0.5 & 0.5 & 0 & 0.125 & 0.5 & 0.125 & 0 \\
\hline$y_{i}$ & 0 & 0 & 0.5 & 0 & -0.125 & 0 & 0.125 & 0 \\
\hline
\end{tabular}

Table 5: Example: vertices and mid-point nodes of a six-noded collapsed quadrilateral quarter-point element $K$.

For this element, the quarter-point map $(x, y)=F_{K}(\xi, \eta)$, and the Jacobian matrix of $F_{K}$ become

$$
\begin{gathered}
x=\frac{1}{8}(1+\xi)^{2}, y=\frac{1}{8} \eta(1+\xi)^{2}, \\
D F_{K}=\left[\begin{array}{cc}
\frac{\partial x}{\partial \xi} & \frac{\partial y}{\partial \xi} \\
\frac{\partial x}{\partial \eta} & \frac{\partial y}{\partial \eta}
\end{array}\right]=\left[\begin{array}{cc}
\frac{1}{4}(1+\xi) & \frac{1}{2} \eta(1+\xi) \\
0 & \frac{1}{8}(1+\xi)^{2}
\end{array}\right],
\end{gathered}
$$

with determinant $\mathbf{J}_{K}=\frac{1}{32}(1+\xi)^{3}$. Parametrized vector functions $\hat{\mathbf{q}}$ associated to some examples of vector functions q defined in $K$ are displayed in Table 3, also showing that $\mathbf{V}_{R T_{1}}(K)$ contains the constant vectors.

The parametric representation of the radial square-root function $w=r^{1 / 2}$ restricted to $K$ is the infinitely smooth function

$$
\hat{w}(\xi, \eta)=\frac{1}{2 \sqrt{2}}(1+\xi)\left(1+\eta^{2}\right)^{1 / 4}
$$

which is plotted in Figure 4 (left side). For $\mathbf{q}=\nabla w$, restricted to $K$, the corresponding parametric representation $\hat{\mathbf{q}}$ is given by the regular vector

$$
\hat{\mathbf{q}}=\frac{1}{4 \sqrt{2}}\left[\begin{array}{c}
\frac{(1+\xi)}{\left(1+\eta^{2}\right)^{\frac{3}{4}}} \\
0
\end{array}\right] \text {. }
$$

The first component of $\hat{\mathbf{q}}$ is represented in the Figure 4 (right side). These facts make the collapsed quadrilateral quarter-point elements more efficient for the representation of radial square-root singularities of type $\sqrt{r}$ than the quadrilateral eight-noded quarter-point elements, as observed in [31] for linear elasticity, and illustrated by the numerical simulations of the next section.
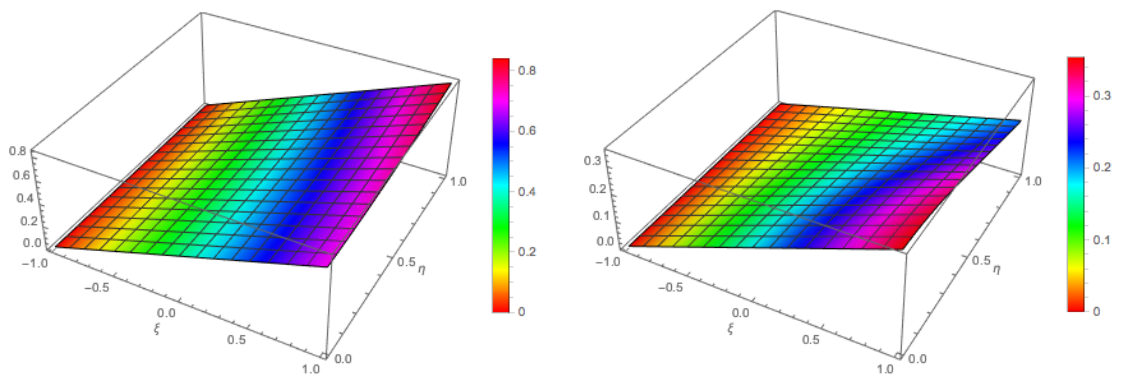

Figure 4: Parametric representation $\hat{w}(\xi, \eta),-1 \leq \xi \leq 1,0 \leq \eta \leq 1$, of $w(r, \theta)=r^{1 / 2}$ restricted to the collapsed quadrilateral six noded quarter point element $K$ (left side), and of the first component of $\hat{\mathbf{q}}$ associated to $\mathbf{q}=\nabla u$ (the second gradient component vanishess) (right side). 

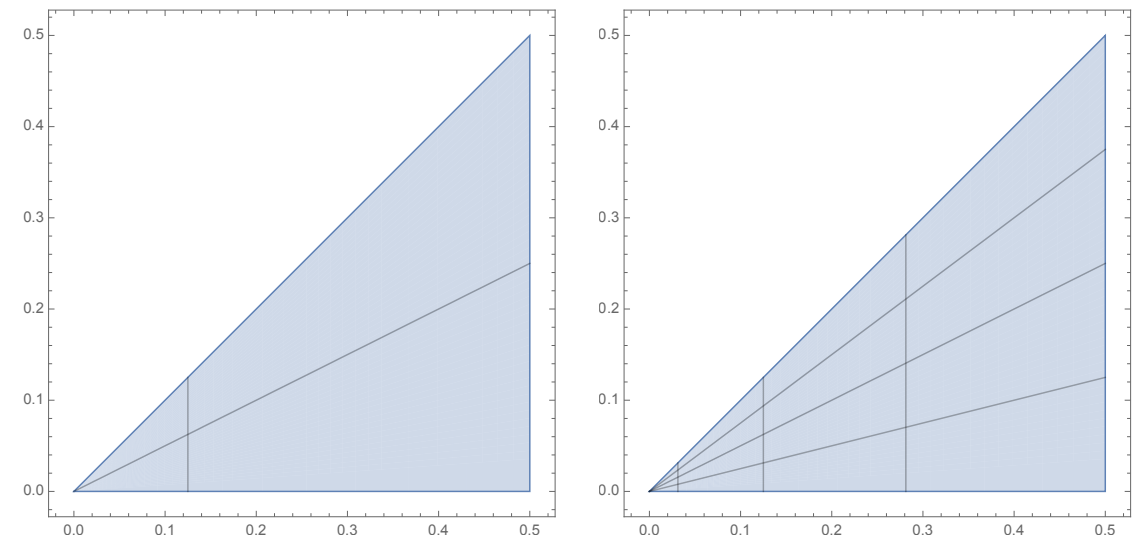

Figure 5: Meshes $\mathcal{T}_{j}^{K}, j=1,2$, obtained by one and two uniform subdivisions of the collapsed quadrilateral quarter-point element $K$.

The first and twice subdivisions of the collapsed quadrilateral quarter-point element $K$ are shown in Figure 5. Since $x$ does not depend on $\eta$, and fixing constant the value of $\xi$, the mapped curve is a vertical line. Otherwise, for constant value of $\eta$, the mapped curve is the strait line $y=\eta x$. Consequently, the resulting meshes $\mathcal{T}_{j}^{K}$, obtained by applying $j$ times the uniform refinement procedure to the original collapsed quadrilateral quarter-point element $K$, are formed by $j$ triangles touching the singular point $(0,0)$, and trapezoidal quadrilateral elements elsewhere. They are graded anisotropic meshes, with growing aspect ratio, as shown in Figure 6 (right side).
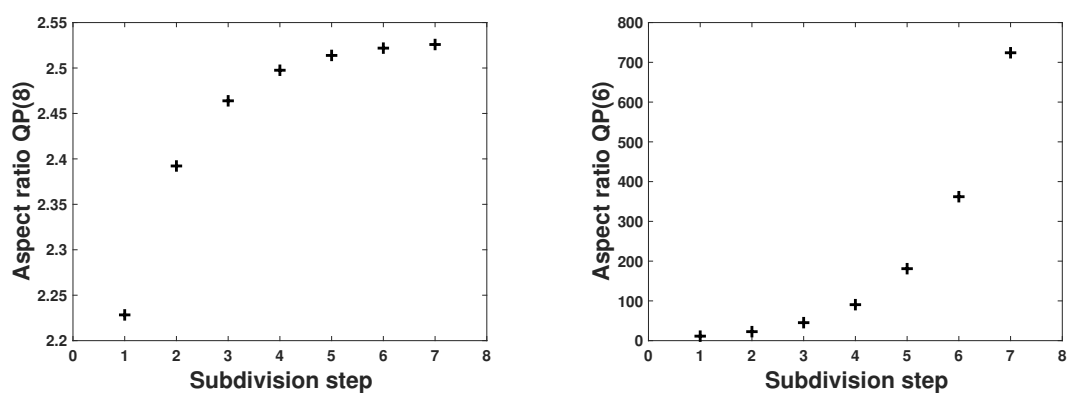

Figure 6: Aspect ration of the quarter-point meshes $\mathcal{T}_{j}^{K}$ obtained by uniform quadrilateral element $K$, with 8 nodes (left side), and the collapsed quadrilateral quarter point element $K$, with 6 nodes.

\section{$5 \quad$ Numerical simulations}

A model problem is considered with $f=0$, and $\Omega=[-0.5,0.5] \times[0,0.5]$, where the exact solution, shown in Figure 7, is the harmonic function

$$
u=2^{1 / 4} \sqrt{r} \cos \left(\frac{\theta}{2}\right)=2^{-1 / 4} \sqrt{x+\sqrt{x^{2}+y^{2}}},
$$

that presents a radial square root singularity at the boundary point $(x, y)=(0,0)$ $(r=0)$, where there is a change from Dirichlet boundary condition $u=0$, for $x<0$, to 
Neumann condition $\nabla u \cdot \boldsymbol{\eta}=0$, for $x>0$. Elsewhere, Neumann boundary condition is enforced. This problem has been used in [32] to compare the performance of different

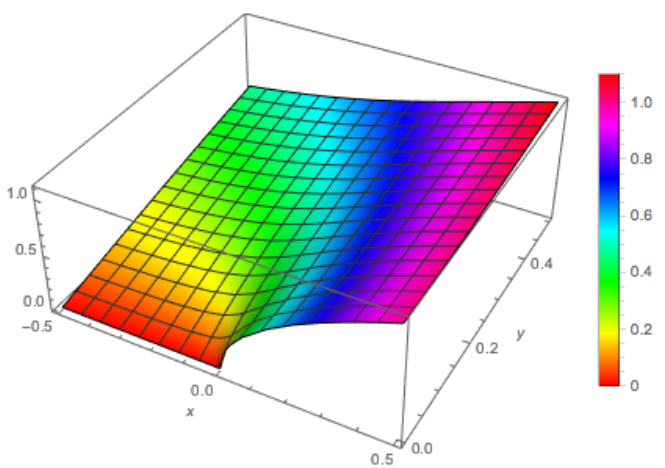

Figure 7: Exact solution $u=2^{1 / 4} \sqrt{r} \cos \left(\frac{\theta}{2}\right)$.

finite element formulations, including continuous, discontinuous, mixed and primal hybrid finite element methods. As expected, the application of $h p$-refinement improves considerably the performance of all methods. The purpose here is to evaluate the efficiency of the mixed method applied to this singular problem when quarter-point elements are used in the vicinity of the singularity.

\subsection{Numerical results with uniform refinement}

Two scenarios shall be considered for the generation of sequences of meshes $\mathcal{T}_{j}, j=$ $0,1,2, \cdots, 5$, by using uniform refinement procedures, as described in Section 4.1. Starting from fixed macro quarter-point elements at the coarse level, they are refined by mapping uniformly refined square meshes on the master element by the corresponding geometric transforms.
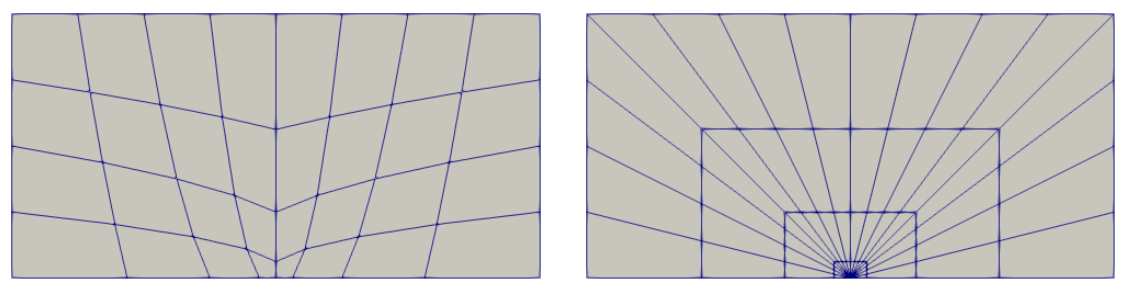

Figure 8: Mesh scenarios: $\mathcal{T}_{2}$ starting with two eight-noded quarter-point elements sharing the singularity point (left side), or starting with four triangular six-noded quarter-point elements.

\section{Mesh scenario 1}

The coarsest mesh $\mathcal{T}_{0}$ is composed of two square elements $K_{0}^{\ell}$, sharing the singular point $(0,0)$ as one of their vertices, and eight-noded quarter-point maps are applied, as described in the example in Section 4.2. The illustration of the mesh $\mathcal{T}_{2}$, obtained by refining twice these coarse elements, is shown in Figure 8 (left side). As already described in the previous section, the resulting sequence of meshes $\mathcal{T}_{j}$ is obtained by 
mapping uniformly refined square meshes on the master element by the corresponding geometric transform of the corresponding father elements $K_{0}^{\ell}$. Consequently, $\mathcal{T}_{j}$ is shape-regular. Furthermore, by their definition, the shape functions are produced by the quarter-point geometric map are present in all the elements. However, only two elements neighboring the singular node $(0,0)$ have a singular mapping.

Having in mind the example discussed in Section 4.2, the exact singular solution $u^{\ell}=\left.u\right|_{K_{0}^{\ell}}$ can be represented back in the master element by smoother functions $\hat{u}^{\ell}(\xi, \eta)=u^{\ell}\left(\mathbb{F}_{K_{0}^{\ell}}(\xi, \eta)\right)$ obtained by composing $u^{\ell}$ with the quarter-point mappings of the two coarse elements $\mathbb{F}_{K_{0}^{\ell}}$. Consequently, the application of the mixed method to solve the model test problem using stable approximation spaces based on $\mathcal{T}_{j}$ are expected to give better convergence rates than using full square uniform meshes. As remarked in the introduction, this behavior is in accordance with the underlying idea of mapped element methods [16, 17], in the context of $H^{1}$ conforming methods for linear elasticity of crack problems, by interpreting the method as a reparametrization of the problem that removes the singularity, the corresponding smoother parametric solution $\hat{u}$, with lower gradients, being able to be accurately solved using approximations based on standard affine uniform partitions in the parametric domain.

Figure 9 presents $L^{2}$-error curves for the approximations of $u$ and $\boldsymbol{\sigma}$ obtained with the mixed method based on $R T_{k}$ and $R T_{k}^{+}$space configurations based on the meshes $\mathcal{T}_{j}, 1 \leq j \leq 5$, with uniform polynomial degree distribution $k=1,2$ (indicated by QP $R T_{k}$ or QP $\left.R T_{k}^{+}\right)$. The mesh size $h_{j}$ are for the associated uniform meshes on the master element, whose images by $F_{K_{0}^{\ell}}$ form the refined meshes $\mathcal{T}_{j}$ restricted to each macro element $K_{0}^{\ell}$. Results for uniform square meshes are also included (denoted by Square $R T_{k}$ ). As expected for this kind of singular solution $u \in H^{\frac{3}{2}-\epsilon}, \forall \epsilon>0$, the convergence
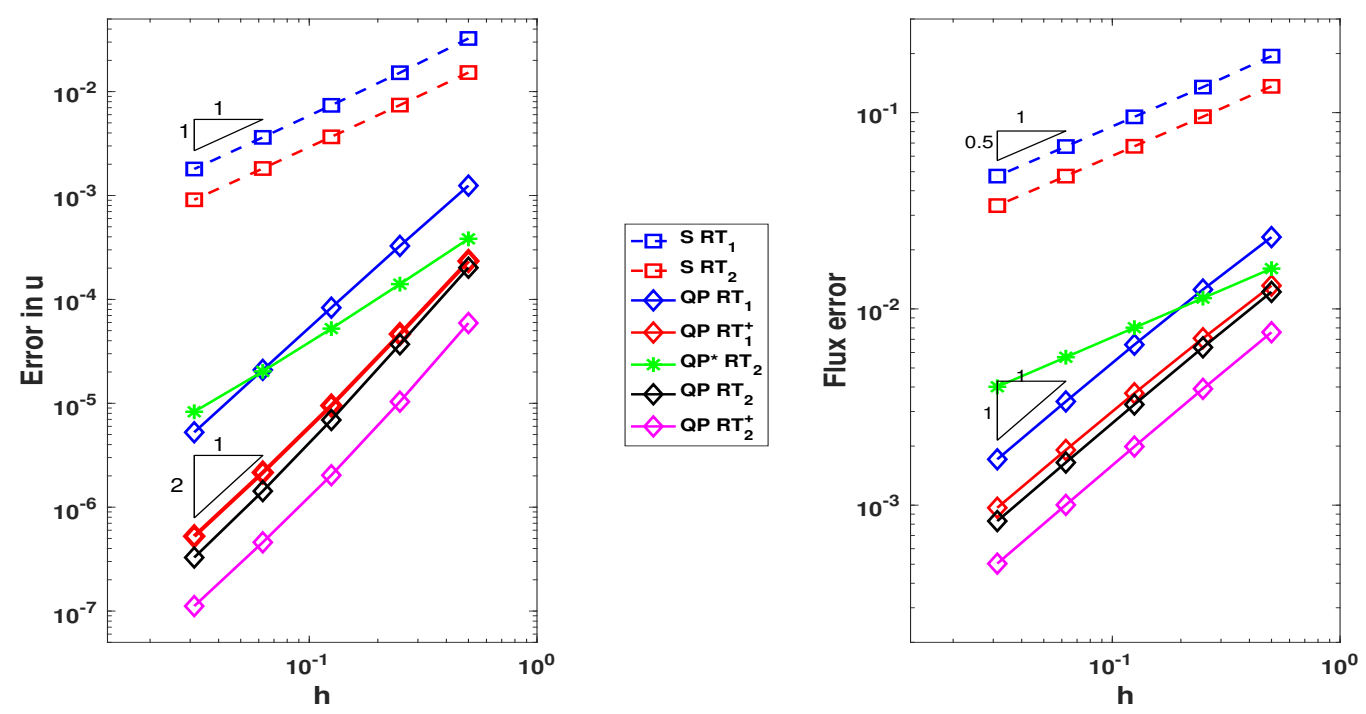

Figure 9: $L^{2}$-erros in $u$ (left side) and in $\boldsymbol{\sigma}$ (right side) using Raviart-Thomas spaces $R T_{k}$, or $R T_{k}^{+}$, with $k=1,2$, based on square meshes (S-dashed lines), on meshes formed by quadrilateral quarter-point elements of 8 nodes (QP-continuous lines), or with just two quadrilateral quarter-point elements of 8 nodes sharing the singular vertex ( $\left.\mathrm{QP}^{*}\right)$. 
of flux and potential approximations given by the application of the $R T_{k}$ space configuration based on uniform square meshes, are limited to rates of order 0.5 and 1.0, respectively, independently of the polynomial degree $k \geq 1$. When quarter-point elements are applied, enhanced approximations are observed, not only by increasing the rates to order 1 for the flux and 2 for the potential, but also by the significant reduction in error magnitudes. The enriched $R T_{k}^{+}$space configurations based on quarter-point meshes give more accurate solutions, specially for the potential variable. For instance, the errors in $u$ using $R T_{2}^{+}$on the most refined mesh $\mathcal{T}_{5}$ is about four orders of magnitude less than using $R T_{2}$ on the square mesh with the same resolution.

Figure 8 also includes plots for the results obtained with $R T_{2}$ space configuration based on meshes where only the two refined quadrilateral elements sharing the singular vertex are mapped by the quarter-point transformation (indicated by $\mathrm{QP}^{*} R T_{2}$ ). Confronted with the simulation for $R T_{2}$ spaces on the full affine square meshes, the introduction of these two quarter-point elements significantly reduces the error magnitudes, but the convergence rates do not improve. This behavior is in accordance with the directions given in [17], where the singularities are localized in a fixed sector, where they are properly parametrized, and approximated by uniform discretizations.

\section{Mesh scenario 2}

A second mesh scenario considers the coarsest mesh $\mathcal{T}_{0}$ composed of 4 triangular elements $K_{0}^{\ell}$ sharing the singularity point $(0,0)$, and six-noded quarter-point maps are applied to them. The meshes $\mathcal{T}_{j}$ are constructed by the uniform refinement procedure applied to $K_{0}^{\ell}$, as described in the example in Section 4.3. The resulting anisotropic mesh $\mathcal{T}_{2}$, obtained by refining twice the coarsest mesh, is shown in Figure 8 (right side).

As in the previous scenario, the exact singular solution $u^{\ell}=\left.u\right|_{K_{0}^{\ell}}$, restricted to each of the four coarse triangular elements, can be represented back in the master element by smoother functions $\hat{u}^{\ell}(\xi, \eta)=u^{\ell}\left(\mathbb{F}_{K_{0}^{\ell}}(\xi, \eta)\right)$ obtained by composing $u^{\ell}$ with the corresponding quarter-point mappings. However, opposed to the mesh scenario 1 , having a limited smoothness improvement, $\hat{u}^{\ell}(\xi, \eta)$ are infinitely smooth in the mesh scenario 2, as has been illustrated in Section 4.3. Consequently, the application of the mixed method to solve the model test problem using stable approximation spaces based on the meshes $\mathcal{T}_{j}$ of scenario 2 are expected to give better convergence rates than using the meshes of scenario 1. Indeed, as confirmed by the error plots of Figure 10, this property is crucial to compensate the effect of mesh anisotropy, and to produce convergence rates of order $k+1$ for flux $\boldsymbol{\sigma}$ and potential $u$ for $R T_{k}$ space configurations, which is typical of standard regular scenarios (shape-regular affine quadrilateral meshes and smooth solutions), and enhanced potential order of convergence $k+2$ occurring for the enriched space configuration $R T_{k}^{+}$. In order to confirm this tendency to higher polynomial degrees, the results in Figure 11 are for a $p$-refinement experiment based on the coarse level $\mathcal{T}_{1}$, clearly showing an exponential error decay as $k$ increases.

\section{$5.2 \quad h p$-Adaptive simulations}

Even though the singular solution being better represented by approximations based on quarter-point elements, for uniformly refined partitions $\mathcal{T}_{j}$, as discussed in Section 5.1, 

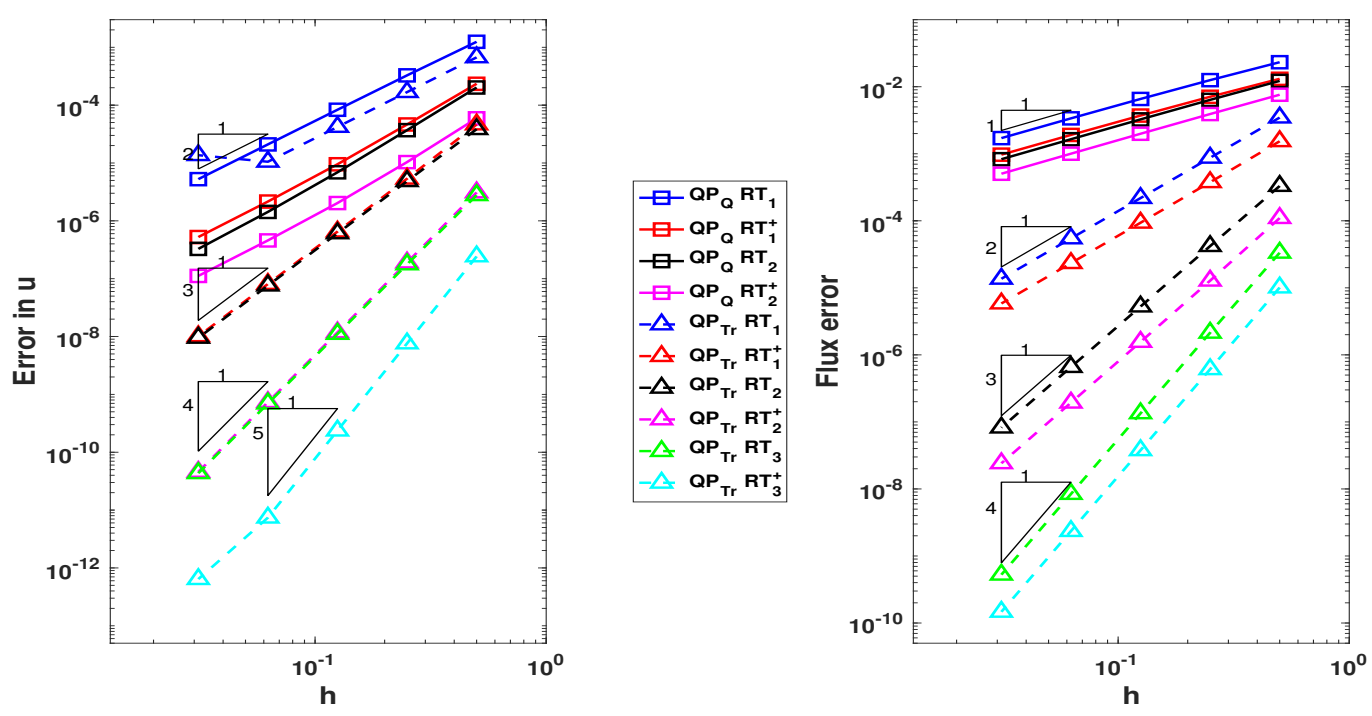

Figure 10: $L^{2}$-errors in $u$ (left side) and in $\boldsymbol{\sigma}$ (right side), versus the mesh width $h$ of the master element, using Raviart-Thomas spaces $R T_{k}$, or $R T_{k}^{+}$, based on quarter-point elements: refining the two quarter-point elements with 8 nodes $\left(Q P_{Q}\right)$ (continuous lines), or the four colappsed quadrilateral quarter-point elements with 6 nodes $\left(Q P_{T r}\right)$.

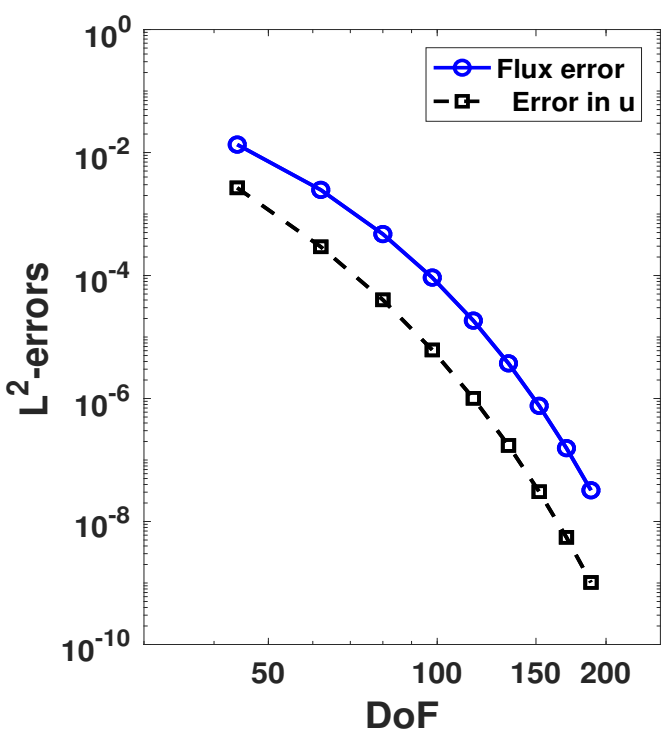

Figure 11: $L^{2}$-errors in $u$ (dashed line $(\mathrm{s})$ ) and in $\boldsymbol{\sigma}$ (continuous line (o)) versus the number of equations of the condensed systems, using Raviart-Thomas spaces $R T_{k}$, $k=1, \cdots, 9$, based on the fixed collapsed quadrilateral quarter-point mesh $\mathcal{T}_{1}$.

the experiments for the mesh scenario 1 , using $R T_{k}$ and $R T_{k}^{+}$space configurations using eight-noded quadrilateral geometric maps, the improvements revealed to be limited to order $k=1$ for the flux, and $k=2$ for the potential variables, already verified for $k=1$. Now the purpose is to explore an $h p$-adaptive strategy, combined with these kind of 
quarter-point elements to enhance the convergence of the approximate solutions.
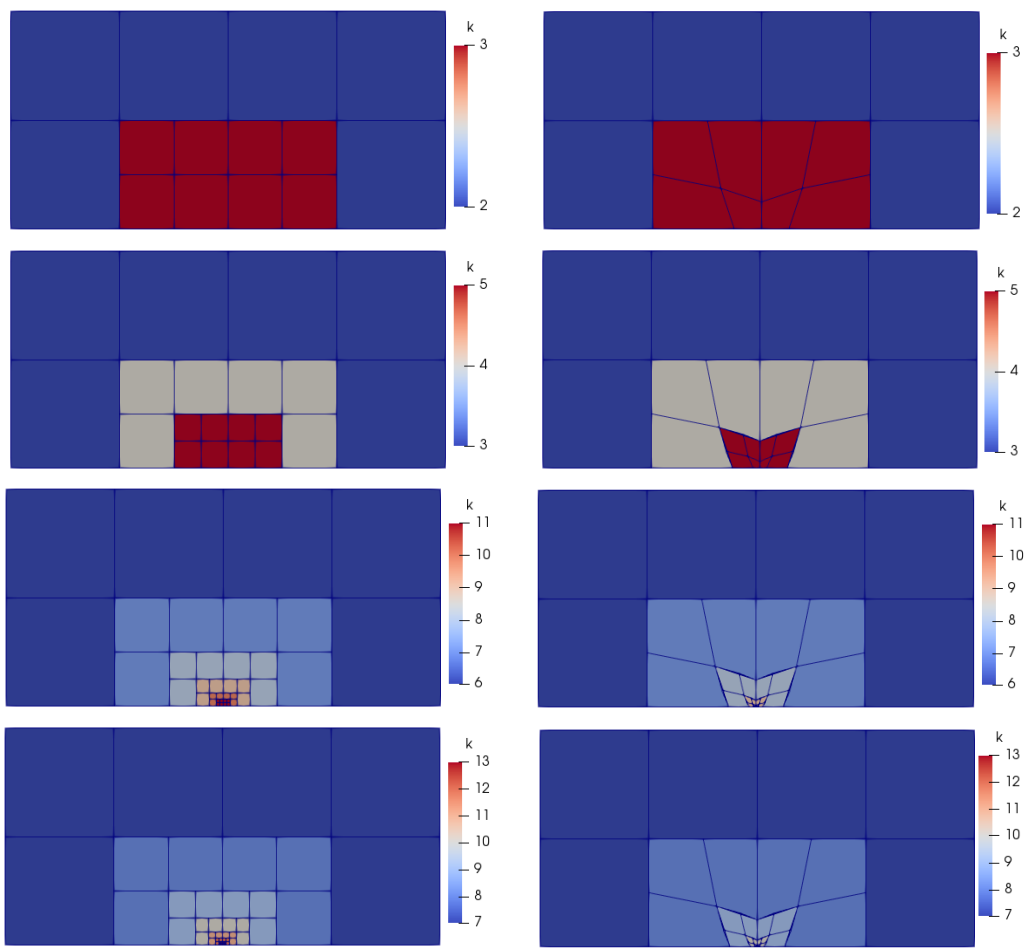

Figure 12: Illustration of the $h p$ meshes. Affine meshes (left side), and quarter-point meshes (right side), at refinement levels 1, 2, 5, and 6 (from top to bottom).

The construction of the $h p$-adaptive meshes is illustrated in Figure 12. Initially, there is a mesh composed of 8 quadrilateral elements with uniform degree $k=2$ distribution. The two quadrilaterals near the singularity are divided into 4 sub-elements and their approximation order are set to $k=3$. Then, the refinement process follows the steps:

1. All elements have their approximation order increased by 1 .

2. The elements near the singularity are divided and their approximation order are increased by 1 .

Two cases shall be compared: one with affine mapping applied to all elements, and the other one for eight-noded quarter-point elements in the central region $\Omega_{C}$. Our purpose is to use this kind of adaptive meshes, for the simulation of the test problem by the mixed formulation using $R T_{\mathbf{k}}$ and $R T_{\mathbf{k}}^{+}$space configurations, where $\mathbf{k}=\left(k_{K}\right)$ refers to the non-uniform polynomial degree distribution used in the construction of the approximation spaces.

Figure 13 shows the calculated $L^{2}$-norms of the dual and primal errors using these sequences of $h p$-adaptive quarter-point curved meshes (continuous lines) versus the number of equations solved after static condensation, using $R T_{\mathbf{k}}$ and $R T_{\mathbf{k}}^{+}$(o) space configurations. For comparison, results for similar square $h p$-meshes (dashed lines), without using quarter-point elements, and for uniform meshes with constant $k=2$ distribution $(+)$ are plotted. The results demonstrate exponential rates of convergence 
when using $h p$-adaptive meshes. However, for the standard $h p$-adaptive method, based on square elements, the results for $R T_{\mathbf{k}}$ and $R T_{\mathbf{k}}^{+}$(o) space configurations are quite the same. Furthermore, they only outperforms the uniform setting with quarter-point elements and constant polynomial degree $k=2$ at the very refined levels. Dramatic error reduction is observed when the $h p$-adaptive strategy is combined with quarterpoint elements in the central region, the accuracy in the primal variable being further improved when the $R T_{\mathbf{k}}^{+}$configuration is applied. Results for square $h p$-meshes, but inverting the polynomial degree distribution $(\diamond)$ are also plotted. This type of degree grading, using low order $k=2$ on the two elements sharing the singularity point, and increasing the degree as the elements are placed away from the singularity, has been suggested in [11], Section 5.2, but for the present model problem the results are less accurate than using the proposed opposite degree grading.

The effect of static condensation is also verified for size reduction of the global system to be solved, being more significant with increasing order of approximation. At the finest level of mesh refinement, the number of condensed equations amounts to more than $94 \%$ of the total number of equations.
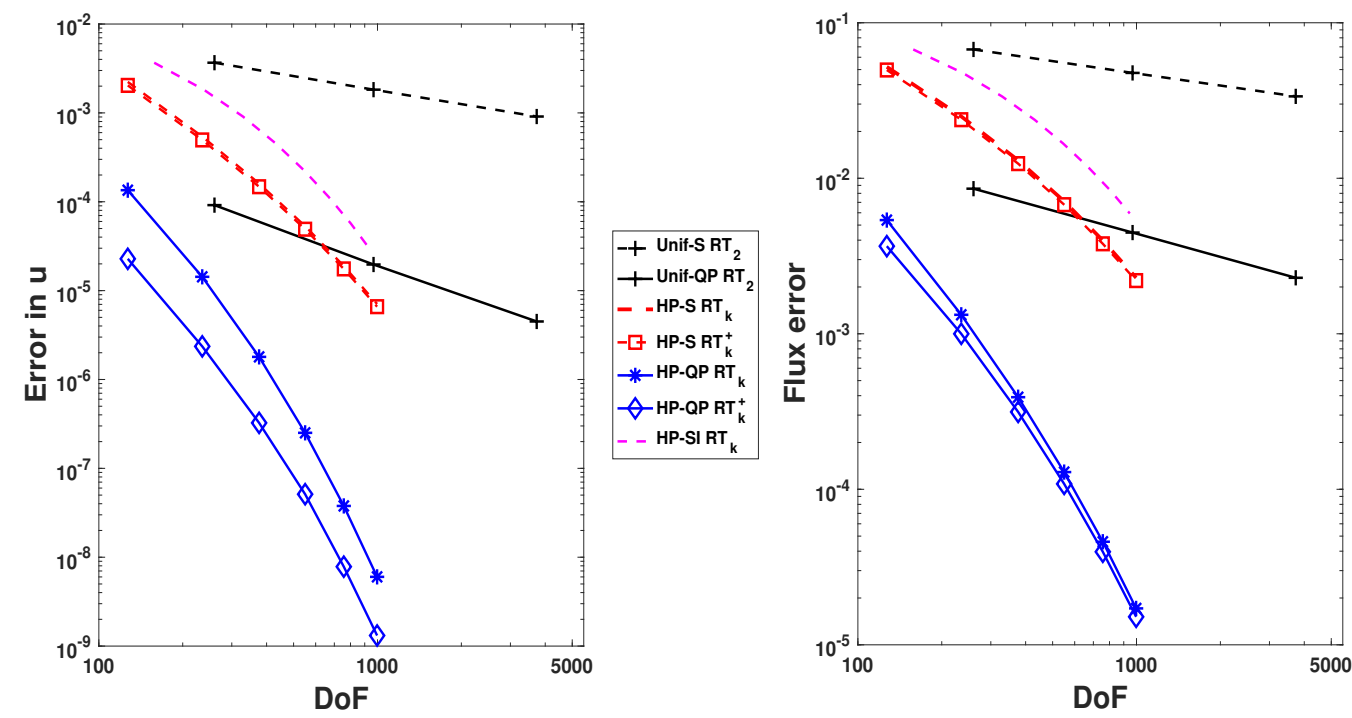

Figure 13: $L^{2}$-errors in $u$ (left side), and in $\boldsymbol{\sigma}$ (right side), using curved quadrilateral meshes (QP) with quarter-point elements (continuous lines), and square (S) meshes (dashed lines), for the mixed formulation based on $h p$-adaptive $R T_{\mathbf{k}}$, and $R T_{\mathbf{k}}^{+}$(circle) space configurations. Uniform simulations $(+)$ are for square and rectangular quarterpoint elements with $R T_{2}$ space distribution. The $h p$-simulations with inverse degree grading $(\diamond)$ are for square meshes $(\mathrm{SI})$.

\section{$6 \quad$ Final conclusions}

This article presents an application of $\mathbf{H}(\mathrm{div})$ mixed approximations, combined with singular geometric map applied to the approximation a problem exhibiting singular behavior caused by a abrupt change in boundary conditions. 
It was shown that the quality of $\mathbf{H}$ (div) approximations of singular problems can be improved dramatically by the use of well chosen geometric maps. This result is an extension to $\mathbf{H}$ (div) conforming spaces of known results when using $H^{1}$ approximation spaces.

When approximating a problem with square root singularity, the well-known quarter point geometric map leads to an increase in the convergence rate of pressure and flux variables, from order 1 and 0.5 when using uniform meshes, to order 2 , and 1 , respectively. It is shown that the combination of quarter point geometric maps with $h-p$ adaptive meshes leads to exponential convergence rates.

Even better approximation properties are obtained by using collapsed quadrilateral elements combined with quadratic geometric maps with quarter point singularity. In this case it is demonstrated that the solution in the inverse image of the geometric map is regular. This property is reflected on the accuracy of the numerical results for flux and pressure: rates of $h$ - convergence equivalent to standard regular contexts, of smooth solutions discretized on uniform affine meshes, and exponential rates of convergence when applying uniform $p$ refinements.

It is probable that the results obtained can be extended to three dimensional simulations of problems with singularities.

\section{Acknowledgements}

The authors P R B Devloo and S M Gomes thankfully acknowledge financial support from FAPESP - Fundação de Apoio à Pesquisa do Estado de São Paulo, Brazil (grant 2016/05144-0), and from CNPq - Conselho Nacional de Desenvolvimento Cientítico e Tecnológico, Brazil (grants 305425/2013-7, 305823-2017-5, and 304029/20130, 306167/2017-4). P R B. Devloo also acknowledges financial support from ANPBrazilian National Agency of Petroleum, Natural Gas and Biofuels (grant 2014/000902).

\section{References}

[1] G. Fix, S. Gulati, G. Wakoff, On the use of singular functions with the finite element method, J. Comput. Phys. 13 (1973) 209-228.

[2] N. Moës, J. Dolbow, T. Belytschko, A finite element method for crack without remeshing, Int. J. Numer. Meth. Eng. 46 (1999) 131-150.

[3] C. Daux, N. Moes, J. Dolbow, N. Sukumar, T. Belytschko, Arbitrary branched and intersecting cracks with the extended finite element method, Int. J. Numer. Meth. Eng. 48 (2000) 1741-1760.

[4] P. Kyoungsoo, J. Pereira, C. Duarte, G. Paulino, Integration of singular enrichment functions in the generalized/extended finite element method for threedimensional problems, Int. J. Numer. Meth. Eng. 78 (2009) 1220-1257. 
[5] A. Yazid, N. Abdelkader, H. Abdelmadjid, A state-of-the-art review of the x-fem for computational fracture mechanics, Appl. Math. Model. 33 (12) (2009) 42694282 .

[6] J. Melenk, I. Babuška, The partition of unity finite element method: Basic theory and applications, Comput. Meth. Appl. M. 139 (1996) 289-314.

[7] R. Barsoum, On the use of isoparametric finite element in linear fracture mechanics, Int. J. Numer. Meth. Eng. 10 (1976) 25-37.

[8] R. Henshell, K. Shaw, Crack tip finite elements are unnecessary, Int. J. Numer. Meth. Eng. 9 (1975) 485-507.

[9] M. Ainsworth, T. J. Oden, A Posteriori Error Estimation in Finite Element Analysis, John Wiley Sons, 2000.

[10] L. Demkowicz, J. Kurtz, D. Pardo, M. Paszynski, W. Rachowicz, A.Zunek, Computing with Hp-Adaptive Finite Elements, Vol. 2: Frontiers Three Dimensional Elliptic and Maxwell Problems with Applications, Chapman \& Hall/CRC, 2007.

[11] C. Schwab, hp-FEM for Fluid Flow Simulation, in: T. Barth, H. Deconinck (Eds.), High-Order Methods for Computational Physics, Vol. 9, Springer, Berlin, Heidelberg, 1999, pp. 325-438.

[12] T. Apel, Anisotropic Finite Elements: Local Estimates and Applications, Advances in Numerical Mathematics, Vieweg+Teubner Verlag, 1999.

[13] M. Farhloul, S. Nicaise, L. Paquet, Some mixed finite element methods on anisotropic meshes, ESAIM 35 (5) (2001) 907-920.

[14] G. Acosta, T. Apel, R. Durán, A. Lombardi, Error estimates for raviart-thomas interpolation of any order on anisotropic tetrahedra, Math. Comput. 80 (273) (2011) 141-163.

[15] H. Li, S. Nicaise, Regularity and a priori error analysis on anisotropic meshes of a Dirichlet problem in polyhedral domains, Numer. Math. 139 (2018) 47-92.

[16] G. Tsamasphyrosm, A. Giannakopoulos, The mapped elements for the solution of cracked bodies, Comput. Meth. Appl. Mech. 49 (3) (1985) 331-342.

[17] M. Chiaramonte, Y. Shen, A. Lew, Mapped finite element methods: High-order approximations of problems on domains with cracks and corners, Int. J. Numer. Meth. Eng. 11 (2017) 864-900.

[18] J. Babuška, B. Andersson, B. Guo, J. Melenk, H. Oh, Finite element method for solving problems with singular solutions, J. Comput. Math. 74 (1-2) (1996) 51-70.

[19] D. Boffi, F. Brezzi, M. Fortin, Mixed Finite Element Methods and Applications, Vol. 44, Springer-Verlag, 2013.

[20] D. Arnold, D. Boffi, R. Falk, Quadrilateral H(div) finite elements, SIAM J. Numer. Anal. 42 (6) (2005) 2429-2451. 
[21] L. Demkowicz, Polynomial exact sequence and projection-based interpolation with application maxwell equations, in: D. Boffi, L. Gastaldi (Eds.), Mixed Finite Elements, Compatibility Conditions and Applications, Springer, Berlin, Heidelberg, 2008, pp. 101-158.

[22] P. Solin, K. Segeth, I. Dolezel, Higher-Order Finite Element Methods, Chapman - Hall/CRC, 2004.

[23] S. Zaglamayr, Hight order finite element methods for electromagnetic field computation, Ph.D. thesis, Johannes Keppler Universität Linz (2006).

[24] D. Siqueira, P. Devloo, S. Gomes, A new procedure for the construction of hierarchical high order hdiv and hcurl finite element spaces, J. Comput. Appl. Math. 240 (2013) 204-214.

[25] D. Castro, P. Devloo, A. Farias, S. Gomes, D. Siqueira, Three dimensional hierarchical mixed finite element approximations with enhanced primal variable accuracy, Comput. Method. Appl. M. 306 (2016) 479-502.

[26] P. Devloo, A. Farias, S. Gomes, D. Siqueira, Two-dimensional $h p$-adaptive finite element spaces for mixed formulations, Math. Comput. Simulat. 126 (2016) 104122 .

[27] P. Devloo, O. Durán, S. Gomes, N. Shauer, Mixed finite element approximations based on 3D $h p$-adaptive curved meshes with two types of $\mathrm{h}(\mathrm{div})$-conforming spaces, Int. J. Numer. Meth. Eng. 113 (7) (2017) 1045-1060.

[28] R. Durán, Mixed finite element methods, in: Mixed Finite Elements, Compatibility Conditions and Applications, Springer-Verlag Berlin Heidelberg, 2008, Ch. 1, pp. $1-44$.

[29] A. Ern, J. Guermond, Finite element quasi-interpolation and best approximation, ESAIM 51 (4) (2017) 1367-1385.

[30] A. Farias, P. Devloo, S. Gomes, D. Siqueira, D. Castro, Two dimensional mixed finite element approximations for elliptic problems with enhanced accuracy for the potential and flux divergence, Comput. Math. Appl. 74 (12) (2017) 3283-3295.

[31] R. Barsoum, Triangular quarter-point elements as elastic and perfectly-plastic crack tip elements, Int. J. Numer. Meth. Eng. 11 (1977) 85-98.

[32] T. Forti, A. Farias, P. Devloo, S. Gomes, A comparative numerical study of different finite element formulations for 2D model elliptic problems: continuous and discontinuous galerkin, mixed and hybrid methods, Finite Elem. Anal. Des. 115 (2016) 9-20. 